\title{
Case series of six patients diagnosed and managed for idiopathic intracranial hypertension at a tertiary institution eye centre
}

\author{
Naa N. Tagoe ${ }^{1}$, Vera M. Beyuo ${ }^{2}$ and Kwesi N. Amissah-Arthur ${ }^{2}$
}

Ghana Med J 2019; 53(1): 79-87 http://dx.doi.org/10.4314/gmj.v53i1.12

\author{
${ }^{1}$ Lions Eye Centre, Korle Bu Teaching Hospital Accra, Ghana \\ ${ }^{2}$ Department of Surgery, School of Medicine and Dentistry, College of Health Sciences, University of Ghana, \\ Legon, Accra, Ghana.
}

Corresponding author: Naa Naamuah Tagoe

E-mail: naanaamuahtagoe@gmail.com

\author{
Conflict of interest: None declared
}

\section{SUMMARY}

Background: Idiopathic Intracranial Hypertension (IIH) occurs secondary to raised intracranial pressure (ICP) of unknown etiology and is diagnosed when all other causes of raised ICP have been excluded. It can leave devastating sequelae such as permanent visual loss, hence the need for timely diagnosis and treatment. Anecdotally, one or two cases of idiopathic intracranial hypertension (IIH) previously presented at the Eye Centre, KBTH yearly. However, six cases were seen within a 6-month period, prompting the need to study the clinical features of IIH in this population. Objective: We aim to evaluate the clinical features of patients presenting with IIH at KBTH.

Methodology: This is a retrospective case series with contemporaneous collection of data of six patients who presented to the Eye Centre (KBTH) between October 2016 and March 2017 with clinical features suggestive of IIH. The patients were evaluated and diagnosed based on clinical judgement as well as using the modified Dandy criteria. Results: All six patients were female and all except one were obese. The age range was 8 to 40 years with median 22.5 years. Symptoms in the 8-year-old were preceded by oral doxycycline for acne treatment. One patient had a history of using oral contraceptive pills prescribed for irregular menses. Clinical features of blurred vision, headache, and papilloedema were relieved with oral acetazolamide.

Conclusion: The upsurge of IIH may be due to the increased incidence of obesity in Ghana. Timely diagnosis and treatment is needed to avoid irreversible blindness.

Funding: None

Keywords: headaches, idiopathic intracranial hypertension, obesity, blindness, raised intracranial pressure.

\section{INTRODUCTION}

Idiopathic intracranial hypertension (IIH) occurs when there is raised intracranial pressure (ICP) of unknown etiology and is diagnosed when all other causes of raised ICP have been excluded. ${ }^{1}$ It was previously known as "Pseudo tumor cerebri" and "benign intracranial hypertension". It is a diagnosis of exclusion. Though previously referred to as benign, it can leave devastating sequelae such as permanent visual loss in $31 \%$ of cases, ${ }^{2}$ hence the need for early diagnosis and intervention. Suggestions have been made that the terms "primary" and "secondary" intracranial hypertension should be considered to describe these two groups of patients: 1) the young obese women with isolated raised ICP and no obvious precipitating factors and 2) patients with isolated raised ICP associated with factors such as endocrine disorders, anemia, obstructive sleep apnea, medications, or cerebral venous sinus stenosis.,
The currently used term "idiopathic intracranial hypertension" reflects the general lack of understanding of the pathophysiology of this disorder.

Diagnosis is made using the modified Dandy criteria:

1) Symptoms and signs of raised ICP i.e. headache, nausea, vomiting, pulsatile tinnitus, transient visual obscurations, papilledema)

2) Absence of localizing signs, except for abducens nerve palsy

3) No identifiable cause for raised ICP on neuroimaging (brain MRI or CT scan)

4) Cerebrospinal fluid (CSF) opening pressure of greater than $25 \mathrm{cmH} 2 \mathrm{O}$, with normal CSF composition

5) No alternate explanation for the raised ICP 
Anecdotally, only one or 2 cases of idiopathic intracranial hypertension (IIH) presented at the Eye Centre, KBTH in a year. However, six cases were seen within a 6-month period, thus prompting the need to study the clinical features of IIH in this population. The prevalence of IIH in sub Saharan Africa is not documented, however there have been case reports from Nigeria and South Africa. ${ }^{1,2}$

There is still a lot that is not known about the exact pathophysiology of IIH. It is usually a diagnosis of exclusion. It may be a diagnostic challenge in low resource settings such as in Sub Saharan Africa. More awareness needs to be created about this disease in Africa and protocols for diagnosis and management that are suitable for our setting established, to achieve good outcomes and prevent permanent visual loss.

The goal of this study was to document the epidemiology of patients presenting with IIH at KBTH. The specific objectives were:

1. To document the presenting symptoms of patients with IIH at KBTH

2. To study the signs of patients presenting with $\mathrm{IIH}$ at $\mathrm{KBTH}$ and

3. To document the demographic features of patients with IIH

\section{METHODS}

This was a retrospective case series with contemporaneous collection of data of six patients who presented to the Eye Centre (KBTH) between October 2016 and March 2017 with symptoms and signs suggestive of idiopathic intracranial hypertension. This retrospective case series conformed to the Declaration of Helsinki. Patients were evaluated, investigated and diagnosed based on clinical judgement as well as using the modified Dandy criteria.

\section{CASE REPORTS}

\section{CASE 1}

A 31-year-old female presented on September 13, 2016 with a 2- week history of severe headaches and blurred vision. She had been diagnosed of hypertension during her last pregnancy 4 years prior and her blood pressure remained persistently high despite treatment. Her last BP checked was $148 / 85 \mathrm{mmHg}$. Two weeks prior to presentation she started experiencing severe global headaches associated with tinnitus and blurred vision and her symptoms were worse in the morning. There was no history of nausea or vomiting.

On examination she weighed 90 kilograms (kg), height was $1.62 \mathrm{~m}$ and her Body Mass Index (BMI) was 34.3. Her blood pressure was 140/90mmHg. Her best corrected visual acuity was 6/9 in both eyes. The anterior segment examination was essentially normal except for the right pupil which reacted sluggishly and left pupil which had a Relative Afferent Pupillary Defect (RAPD). Her colour vision was moderately impaired $(11 / 20$ on the right and $12 / 20$ on the left using the Ishihara chart). The intraocular pressures were $17 \mathrm{mmHg}$ and $16 \mathrm{mmHg}$ in her right and left eyes respectively and examination of the optic discs revealed marked papilloedema (Frisen stage 4).

Cranial computerized tomography (CT) scan was reported as normal. Our clinical diagnosis was Papilloedema secondary to possible Idiopathic Intracranial hypertension. She was commenced on oral acetazolamide 500mg 4 times daily and referred to a dietician for weight reduction. 4 weeks post treatment her visual acuity had not improved. The patient revealed that she had stopped taking acetazolamide after 2 weeks because it made her feel unwell.

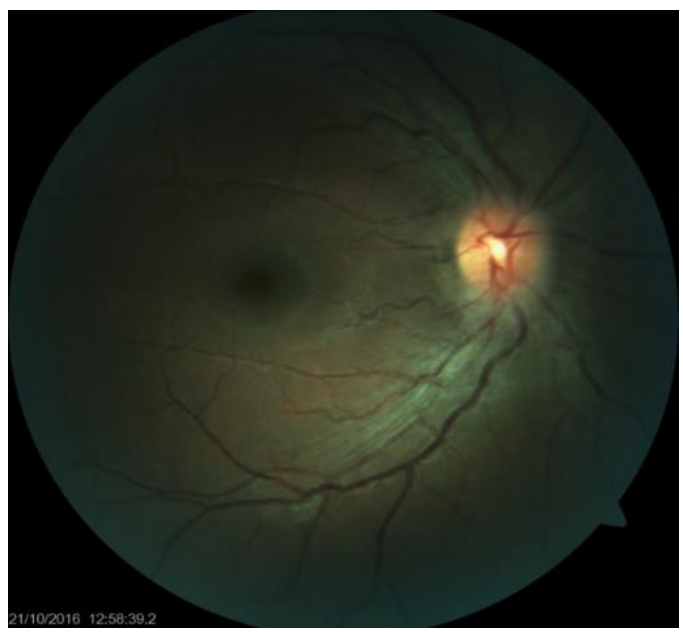

Case 1a Elevation of the entire nerve head, obscuration of all borders and peripapillary halo (RE)

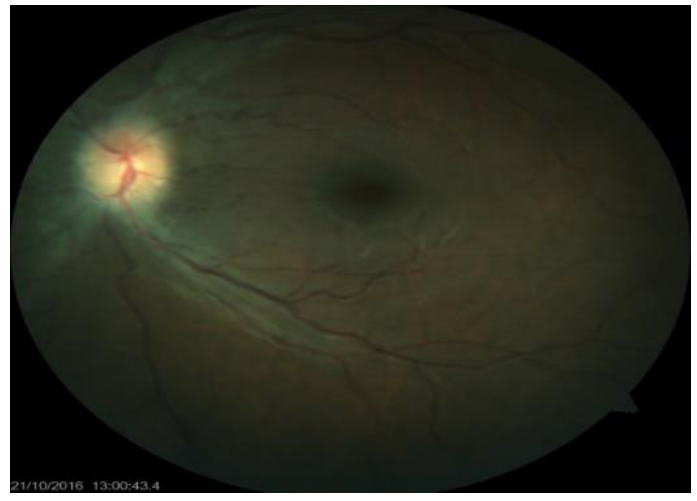

Case 1b Elevation of the entire nerve head, obscuration of all borders and peripapillary halo (LE) 
She was counseled on compliance and the dose of acetazolamide was reviewed to $250 \mathrm{mg}$ twice daily. By the $12^{\text {th }}$ week the headaches and tinnitus had resolved. Her blood pressure (BP) was $110 / 80 \mathrm{mmHg}$ and her weight had reduced to $87 \mathrm{~kg}$, with a BMI of 33.2 . In addition, her best corrected visual acuity had improved to $6 / 6$ in both eyes, her colour vision had returned to normal and the papilloedema had resolved.

\section{CASE 2}

An 8- year- old girl presented on $14^{\text {th }}$ September, 2016, with a 4-month history of recurrent headaches and 3week history of diplopia. She had been on oral doxycycline (a derivative of tetracycline) for acne treatment 2-3 weeks prior to the onset of her symptoms. When the headaches started she reported to a couple of health facilities and had been placed on different analgesics. It was when eye deviation was noticed three weeks prior to presentation that the mother was advised by a doctor relation to do a cranial MRI after which they reported to our centre. On examination, the child was found to be obese. Her weight was $46 \mathrm{~kg}$, height $1.3 \mathrm{~m}$, with a BMI of 27.2.
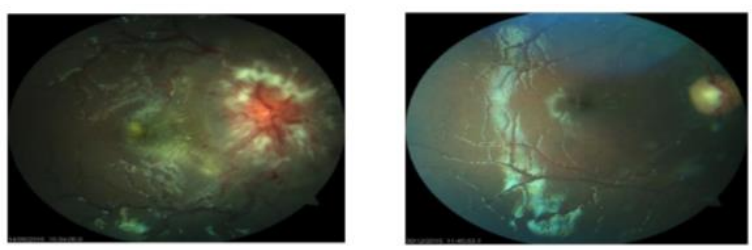

Case 2a severely swollen disc, dome-shaped protrusions, peripapillary haemorrhages with macula exudates (RE). Before and 3 months after photos
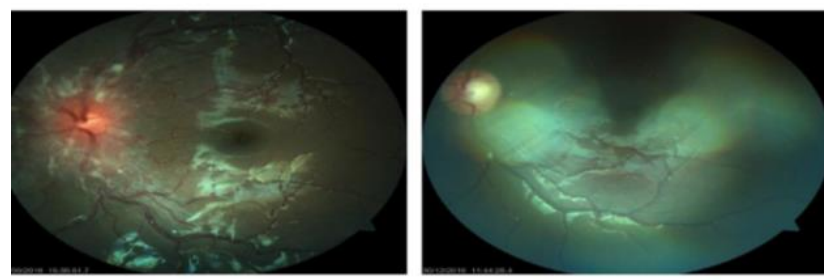

Case 2b Severely swollen disc, dome-shaped protrusions, peripapillary haemorrhages with macula exudates (RE). Before and 3 months post treatment

Her blood pressure- $100 / 60 \mathrm{mmHg}$. The best corrected visual acuity was 6/36 in both eyes (moderately impaired) and she had a right exotropia (divergent squint) of 20 prism diopters. Fundus examination revealed stage 5 (Frisen staging) disc swelling/papilloedema with a macula star. A lumbar puncture was done but the opening pressure could not be measured due to the unavailability of a manometer. MRI done showed a swollen optic nerve.
Child was referred to dieticians for weight reduction and started on oral acetazolamide, an initial dose of $375 \mathrm{mg}$ tid, and stepped up after one week to 500mg tid. On her $3^{\text {rd }}$ clinic visit, the headaches had subsided, diplopia had resolved and visual acuity was 6/9 in right eye (RE) and $6 / 6$ in left eye (LE).

She complained of paraesthesia, hence the morning dose of acetazolamide was tapered to $250 \mathrm{mg}$. At her last consultation, 3 months since her initial presentation her best visual acuity was $6 / 6$ in both eyes, the squint had resolved and her optic nerves were normal. She is currently on oral acetazolamide $125 \mathrm{mg}$ daily and still being followed up.

\section{CASE 3}

A 20-year-old female university student presented on October 18, 2016 with a month's history of poor vision in both eyes, worse in the right. She started experiencing black outs initially and later developed severe headaches that were frontal, radiating to the eyes. She reported to a tertiary facility where a Cranial CT scan was done and reported as normal. She was subsequently put on some eye care supplements. However when there was no significant improvement in her symptoms she reported to a private eye clinic where she was commenced on oral acetazolamide $250 \mathrm{mg}$ bd and referred to the Eye Centre, Korle $\mathrm{Bu}$ Teaching Hospital for further management.

On examination she weighed $124 \mathrm{~kg}$ and her blood pressure was $149 / 96 \mathrm{mmHg}$. Her best corrected visual acuity was 6/36 in RE and 6/12 in LE. There was normal ocular alignment with no ophthalmoplegia. Fundus examination showed stage 5 papilloedema (Frisen grading), haemorrhages, exudates, macula oedema with macula star. A lumbar puncture was done and the opening pressure was $35 \mathrm{cmH}_{2} \mathrm{O}$. Test results of CSF biochemistry and bacteriology were negative.

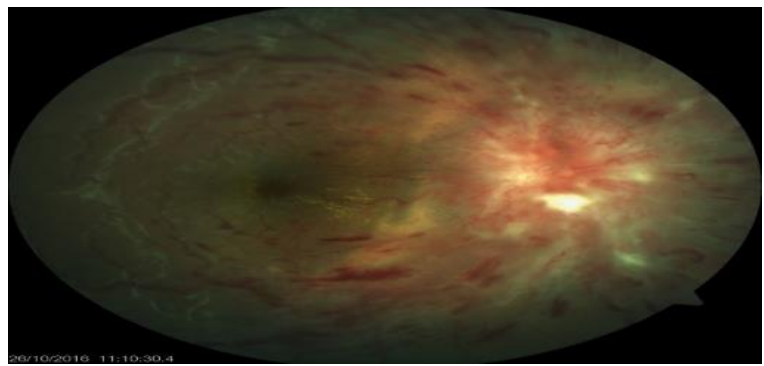

Case 3a Severely swollen disc, obscuration of all vessels, complete obliteration of cup, retinal haemorrhages and macular exudates (RE) 


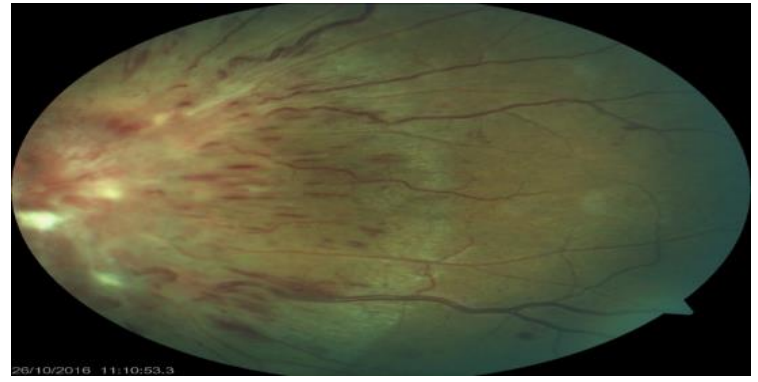

Case 3b. Severely swollen disc, obscuration of all vessels, complete obliteration of cup, retinal haemorrhages and macular exudates (LE)

The patient was referred to see the dieticians for weight reduction. The dose of Acetazolamide was increased initially to $500 \mathrm{mg}$ three times daily and a week later to $500 \mathrm{mg} 6$ hourly. On week two review visit, her weight had dropped to $121 \mathrm{~kg}$, the symptoms of headache had improved, and visual acuity had improved to $6 / 24$ in the right eye. By one-month review, visual acuity in the right eye had improved to $6 / 18$ while that of the left eye remained 6/12. The patient returned to school which was in another region and has since been lost to follow up. She was however contacted via phone and stated that she was doing well.

\section{CASE 4}

A 21-year- old female was referred from the neurosurgical unit on $20^{\text {th }}$ October 2016, with a diagnosis of IIH. She presented with headaches, nuchal rigidity and horizontal diplopia. On examination, she was obviously obese. Her weight was $104 \mathrm{~kg}$, height of 1.76 , with BMI of 33.57. Her best corrected visual acuity was 6/6-1 in both eyes and she had bilateral esotropia.

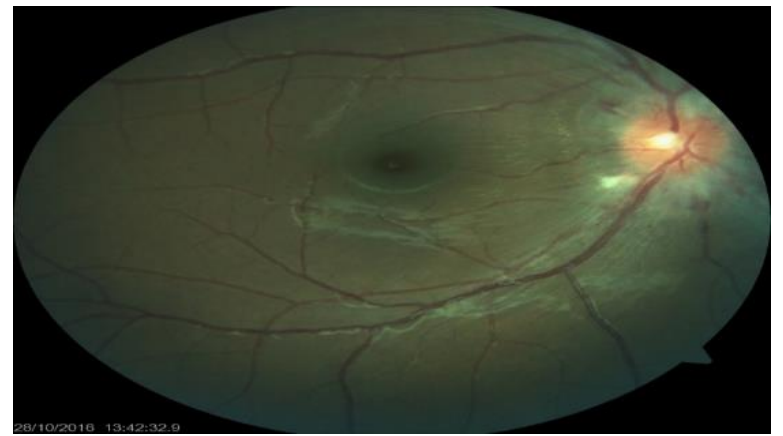

Case 4a Disc swelling, peripapillary haemorrhages, cotton wool spots (RE)

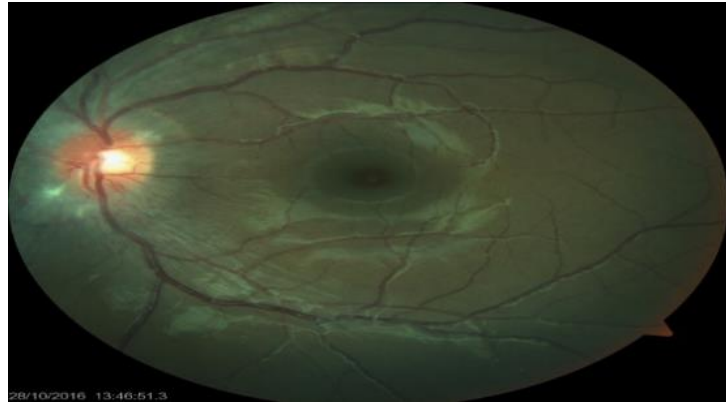

Case 4b Disc swelling, peripapillary cotton wool spots (LE)

Fundus examination revealed bilateral disc oedema (papilloedema), haemorrhages and cotton wool spots. The macula was normal on both sides. The CSF opening pressure was $55 \mathrm{cmH}_{2} \mathrm{O}$. She was referred to dieticians for weight reduction and continued on oral acetazolamide $500 \mathrm{mg}$ bd and oral furosemide $80 \mathrm{mg}$ bd which had been started by her physicians. This patient has been lost to follow up.

\section{CASE 5}

A 24-year-old female presented with a 3-week history of headache and blurred vision. She had associated symptoms of photophobia, diplopia which was binocular, constant and horizontal as well as transient visual obscurations (TVOs). She also had tinnitus, nausea and neck pain. The patient had a history of irregular menses for which she was being managed by the gynaecologists and had been placed on oral contraceptive pills (OCP). The symptoms started around the same time she commenced the OCPs. On examination her weight was $100 \mathrm{~kg}$ and height $1.73 \mathrm{~m}$, with BMI 33.4. Her blood pressure was $120 / 72 \mathrm{mmHg}$. The best corrected visual acuity was $6 / 6$ in the right eye and 6/9 in the left eye.

Colour vision was normal at 11/12 in both eyes using Ishihara colour plates. Examination of the optic discs revealed Grade 3 papilloedema. Cranial CT scan showed small ventricles but was otherwise normal. A lumbar puncture was done but the opening pressure was not measured. Results of her CSF biochemistry and bacteriology were negative. 


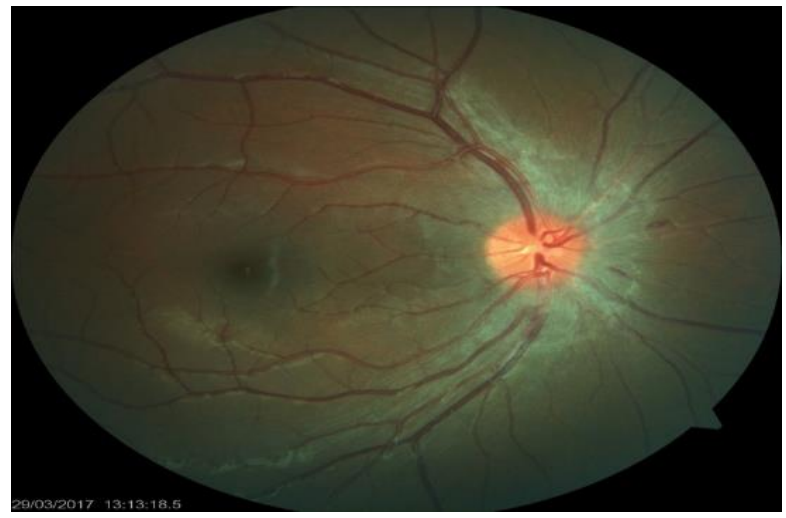

Case 5a. Obscuration of major vessels inferiorly, peripapillary haemorrhages (RE)

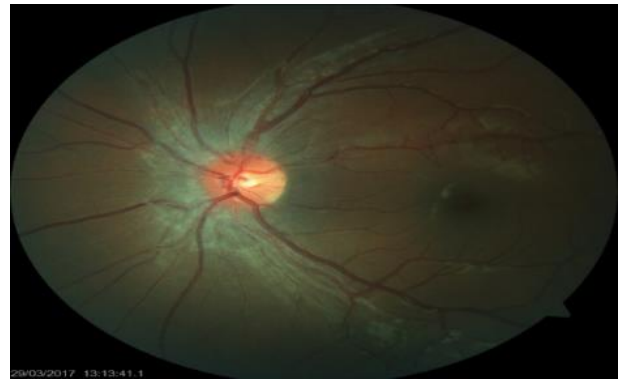

Figure 5b Obscuration of major vessels superiorly, peripapillary halo (LE)

She was initially seen by physicians who started her on IV methylprednisolone, IV Ceftriaxone, oral acetazolamide $250 \mathrm{mg}$ tid and 2 doses of IV mannitol. She was subsequently referred to the Eye Centre, KBTH where upon complaints of persistent headache, the dose of acetazolamide was increased to $250 \mathrm{mg}$ qid. A week later she still had headaches hence the dose of acetazolamide was further increased to $500 \mathrm{mg}$ tid and referred to dieticians for weight loss. By week 3, the headaches, diplopia and blurred vision had subsided. However, she developed an urticarial rash hence the dose of acetazolamide was reduced to $500 \mathrm{mg}$ bd interspersed with oral furosemide $40 \mathrm{mg}$ bd which was well tolerated.

\section{CASE 6}

A 40-year-old woman presented with a prolonged history of headache and a week history of floater in her left eye. She reported to a private eye clinic where she was examined and subsequently referred to our facility for management. Prior to the appearance of the floater she had been experiencing severe headaches associates with vomiting and tinnitus. She frequently took amoxicillinclavulanic acid for recurrent tonsillitis, otherwise there was no other drug history of significance. On examination her BMI was 40.98 (weight was $97.2 \mathrm{~kg}$, height $1.54 \mathrm{~m}$ ), and her BP was $138 / 85 \mathrm{mmHg}$

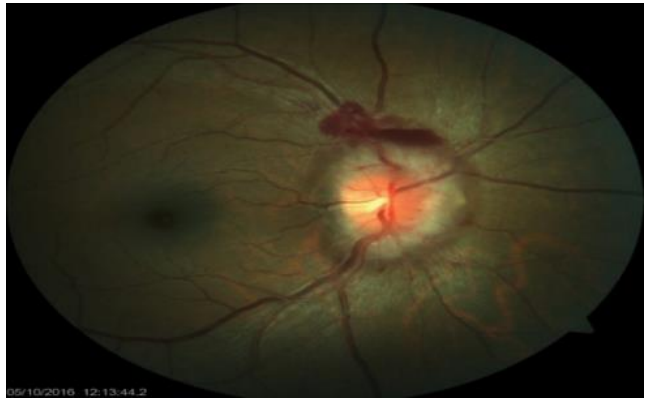

Case 6a Obscuration of major vessels inferiorly, circumferential blurring of disc margins, superior peripapillary haemorrhage $(\mathrm{RE})$

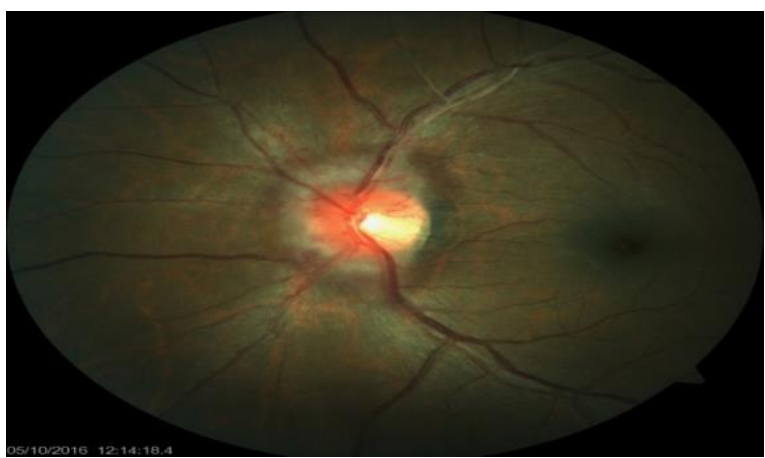

Case 6b. Obscuration of major vessel inferiorly, circumferential blurring of disc margins (LE)

Her visual acuity was counting fingers at 4 metres in both eyes, best corrected to 6/6 with glasses. Colour vision was normal; 15/15 (Ishihara colour vision chart) and funduscopy revealed moderate papilloedema. (Frisen grade 3). Cranial CT scan showed an empty sella. She was referred to the dieticians for weight loss and commenced on oral acetazolamide $250 \mathrm{mg}$ tid. Her symptoms of headache, vomiting and tinnitus abated on this dose.

\section{DISCUSSION}

Several studies have shown that IIH is more common in women and obese individuals. ${ }^{5-8}$ Among women aged 20-44 years who are $20 \%$ or more above ideal body weight, the incidence increases to approximately $19 / 100,000$, about 20 times the incidence in the general population and similar to the incidence of multiple sclerosis. ${ }^{5,9}$ Prior to the period of this report, very few cases of suspected IIH were seen in the department. This could be attributable to the improvement in diagnostic capabilities in our centre or due to the increasing incidence of obesity in the developing world of which Ghana is inclusive. The prevalence of IIH in sub Saharan Africa is not documented, however there have been case reports from Nigeria and South Africa. ${ }^{10,11}$ 
A study in Libya between 1982 and 1989 with 81(76 female, 5 male) patients diagnosed with IIH revealed the following annual incidence rates:

2.2 cases $/ 100,000$ population (total population) 12 cases/100,000 women aged $15-44$ years. ${ }^{12}$

All six patients in this study were female and all except one were obese. This is in line with several other studies that observed that IIH is more common in women and obese individuals. ${ }^{5-8}$ In a recent multicenter case-control study of newly-diagnosed women with IIH compared to women who were newly-diagnosed with other neuroophthalmologic disorders, it was found that higher body mass index is associated with a greater risk of IIH. ${ }^{13}$ The same study also showed that even non-obese patients were at greater risk for IIH if they had a recent moderate weight gain (5-15\%).

Thus, it is likely that the prevalence of IIH in the developed world will rise in parallel with that of obesity. ${ }^{13}$

One of our patients was an eight-year-old female. With the exception of this child, all the other patients were between ages 20 to 40 years. Despite a high predilection for obese young women, IIH can occur in children, older adults, and in non-obese persons of either sex. ${ }^{14,15}$

In children, the annual incidence for symptomatic disease is $0.9 / 100,000 .{ }^{14}$ It was previously thought that there was no sex predilection in IIH among children however Balcer et al found that prevalence of IIH increased with age. He found that children with IIH aged 3 to 11 years, $50 \%$ were girls, 12 to 14 years of age, $88 \%$ were girls, and in children older than 15 years, $100 \%$ were girls. ${ }^{16}$

The underlying pathogenesis of IIH is uncertain however raised ICP is a uniform characteristic feature. It appears that changes in the volume of blood, CSF and brain tissue influence intracranial pressure. IIH therefore likely represents a disorder of CSF regulation, potentially through CSF over secretion or impaired drainage. The symptoms and signs in the only paediatric case in this case series was a preceded by a history of taking doxycycline for acne treatment. Doxycycline is a wellrecognized precipitant of idiopathic intracranial hypertension. ${ }^{17}$ Case 5 also had a history of using oral contraceptive pills which had been prescribed for treatment of irregular menses.

Factors such as medications (tetracycline and its derivatives, cyclosporine, lithium, nalidixic acid, nitrofurantoin, oral contraceptives, levonorgestrel and tamoxifen), endocrine abnormalities (such as corticosteroid withdrawal, anabolic steroids, excessive growth hormone, and thyroid disease), vitamin A excess
${ }^{18,19}$ or deficiency, and systemic conditions (such as pregnancy, menstrual irregularities, polycystic ovarian syndrome, anaemia, and obstructive sleep apnoea). ${ }^{20,21}$ have been found in some studies to be associated, precipitate or worsen IIH.

All 6 cases in this series experienced headaches. This is corroborated by studies by Wall $\mathrm{M}$ et al and Giuseffi $\mathrm{V}^{23}$ who reported headache as the commonest presenting symptom. Headache, though the most common symptom at presentation, ${ }^{22,23}$ is less likely to be reported by men than by women. ${ }^{24}$ The headache of IIH is typically pulsatile, global, worse after waking, and exacerbated by manoeuvres that increase intracranial pressure (ICP). Features consistent with migraine, including unilateral throbbing, with nausea and photophobia have also been reported. ${ }^{26}$

In many cases, patients might also have a coexisting primary headache disorder, such as migraine or tension headache ${ }^{25}$ and thus the headache does not always respond to treatments that decrease ICP.

Case 6 had a prolonged history of headaches spanning over a year. Considering the fact that her disc did not show signs of chronic papilloedema or atrophic changes, it is possible that she had a primary headache disorder. It has been reported that back pain, neck pain and radicular pain frequently occur ${ }^{27}$ and neck pain was reported in two of our cases. Papilloedema is the most common sign at presentation. It may be associated with peripapillary haemorrhages, exudates, and macular oedema. Papilloedema commonly results in transient ischaemic obscurations (TVOs) which are due to transient ischaemia of the swollen optic nerve head. TVOs can present as brief episodes of monocular or binocular vision loss. Transient visual obscurations were experienced by 2 out of 6 of our patients (33.3\%). This is lower than the $68 \%$ reported by Giuseffi et al. ${ }^{23}$ This may be due to the fact that most of them may not have understood the symptom of TVO and interpreted it as blurred vision.

Fifty percent of our patients reported horizontal diplopia. This is not a surprising finding since diplopia has been found to occur in one-third to two-thirds of patients with IIH at presentation. ${ }^{28}$ It tends to be binocular and horizontal, as a consequence of abducens nerve palsy, and typically resolves with normalization of ICP.

Untreated papilloedema can progress to irreversible vision loss and secondary optic atrophy in up to $31 \%$ of patients ${ }^{28}$ and this is more likely to occur in men, ${ }^{8}$ black patients ${ }^{7}$ and those with a fulminant onset of $\mathrm{IIH}^{29}$ Visual loss ranged from none to moderately severe in our 
patients. Pulsatile tinnitus has also been reported and this was experienced by three out of six $(50 \%)$ of our patients.

In developing countries diagnosis is particularly a challenge for several reasons. The first reason is failure to recognize the symptoms and suspect the disease. In case 2 , the patient was an 8 year old who had been experiencing the symptoms of headaches for 4 months during which she had attended health facilities. It was only when she started experiencing diplopia that she was seen at a facility where a Cranial MRI was requested and the parents decided to report to the Eye Centre, KBTH for a second opinion. It was a similar occurrence in most of the patients presenting to us. Majority were diagnosed after 2 weeks of onset of symptoms.

On presentation, it is essential to measure the patient's blood pressure to exclude malignant hypertension. ${ }^{11}$ None of our patients had severe or malignant hypertension.

The initial weight must also be checked and documented. Optic nerve function must be assessed by testing visual acuity, pupillary responses and colour vision. The pupils should be dilated for optic nerve head and macula examination. The level of papilloedema should be documented as this helps in follow up. This has been made easier with the use of the Optical Coherence Tomography (OCT) which accurately quantifies the degree of optic disc swelling. ${ }^{30}$ Where not available the Frisen scale can be used to quantify degree of papilloedema. The Frisen scale was used to grade the level of papilloedema in our patients.

Brain imaging is key in excluding space-occupying lesions and other causes of raised ICP as such as hydrocephalus and cerebral venous thrombosis, to make the diagnosis of IIH. ${ }^{1} \mathrm{MRI} / \mathrm{MRV}$ is preferable to CT scan in making a diagnosis. Imaging abnormalities such as empty sella or flattening of the pituitary, tight subarachnoid spaces, flattening of the posterior globe, protrusion of the optic nerve head, enhancement of the prelaminar portion of the optic nerve head, distension of the optic nerve sheath, and vertical tortuosity of the optic nerve are associated with raised ICP are commonly observed in IIH patients. ${ }^{31,32}$ All our patients had neuroimaging however only one did an MRI scan which is the preferred form of imaging to diagnose $\mathrm{IIH}^{32}$

Lumbar puncture plays a significant role in the management of patients with IIH. It is both diagnostic and therapeutic. In our setting however performing a lumbar puncture and measuring opening pressure can be a challenging task for ophthalmologists, as they do not perform the procedure themselves but have to refer patients to the neurology unit for the procedure The lack of a well-established rapport between the two departments often leads to delays in getting the lumbar puncture with opening pressure measurement done. Other factors include lack of appropriate sized lumbar puncture needles, difficulty in accessing manometers and a deficiency of medical personnel who are conversant with the use of manometers to measure CSF pressure. Only 2 out of our 6 patients had their CSF opening pressures measured. The goals of management are to improve the symptoms and preserve vision. It is important to establish at the beginning whether there is any precipitant or contributing factor, and remove it.

Our $5^{\text {th }}$ patient was on oral contraceptive pills at the time of presentation and this was discontinued immediately. In the case of our paediatric patient, the doxycycline had already been stopped prior to presentation. Obesity or recent weight gain are well known associations of $\mathrm{IIH}^{13}$ It has been observed that even a modest degree of weight loss $(5-10 \%)$ is usually required for improvement in symptoms and signs. ${ }^{33-36}$

\section{Drug treatment}

Acetazolamide, a potent enzyme inhibitor of carbonic anhydrase, is currently considered the mainstay of pharmacological management in IIH. It works by impeding the activity at the choroid plexus reducing CSF secretion. Piper et al, in a recent Cochrane systematic review identified two randomised control trials for the use of acetazolamide in $\mathrm{IIH}^{37}$ Dosage is usually started at $1-2 \mathrm{~g} /$ day in divided doses. Most patients cannot tolerate high doses due to unpleasant side effects such as lethargy, nausea, altered sense of taste and paraesthesia. All of our patients were started on oral acetazolamide.

The dose range was $500 \mathrm{mg}-2 \mathrm{~g} /$ day. Doses had to be altered to suit the patient's tolerability to acetazolamide. Case 5 experienced complete alleviation of symptoms on $1.5 \mathrm{~g} /$ day on acetazolamide however developed an urticarial rash for which dose was reduced to $1 \mathrm{~g} /$ day in 2 divided doses interspersed with 2 doses of furosemide $40 \mathrm{mg}$. Topiramate has been reported to be efficacious in relieving headache caused by $\mathrm{IIH} ;{ }^{23}$ however none of our patients was put on it.

\section{Surgery}

Surgical procedures are usually required in patients with a fulminant onset of disease or when other treatments have failed to prevent progressive visual loss. Options include optic nerve sheath fenestration or a CSF diversion procedure, such as ventriculo/lumbo-peritoneal shunting. Ventriculo/lumbo-peritoneal shunting produces a rapid reduction in ICP, often with a subsequent improvement in symptoms and signs, particularly headaches. ${ }^{38,39}$ Surgery was also not indicated in any of our patients. 


\section{CONCLUSION}

In conclusion, IIH can no longer be described as a rare disease in our part of the world. As the prevalence of obesity increases, ${ }^{12}$ clinicians must be prepared to see more cases of IIH. It is important to have a high index of suspicion and those at the primary health care level need to be able to suspect the diagnosis and refer promptly for treatment due to the risk of permanent visual loss.

\section{REFERENCES}

1. Friedman DI, Liu GT, Digre KB. Revised diagnostic criteria for the pseudotumor cerebri syndrome in adults and children. Neurology 2013; 81:1159-65.

2. Digre $\mathrm{KB}$, Corbett JJ. Idiopathic intracranial hypertension (pseudotumor cerebri): a reappraisal. Neurologist. 2001; 7:2-67.

3. Friedman DI, Jacobson DM. Idiopathic intracranial hypertension. J Neuroophthalmol. 2004; 24:138145.

4. Digre KB. Three current controversies in idiopathic intracranial Neuroophthalmology.2009; 33:93-99.

5. Durcan FJ, Corbett JJ, Wall M. The incidence of pseudotumor cerebri: population studies in Iowa and Louisiana. Arch Neurol. 1988; 45:875-877.

6. Radhakrishnan K, Ahlskog JE, Cross SA, Kurland LT, O'Fallon WM. Idiopathic Intracranial Hypertension (pseudotumor cerebri): descriptive epidemiology in Rochester, Minn, 1976 to 1990.Arch Neurol. 1993; 50:78-80.

7. Bruce BB, Preechawat P, Newman NJ, Lynn MJ, Biousse V. Racial differences in Idiopathic Intracranial hypertension. Neurology. 2008; 70:861867.

8. Bruce BB, Kedar S, Van Stavern GP, Monaghan D, Acierno MD, Braswell RA, Preechawat P, Corbett JJ, Newman NJ, Biousse V. Idiopathic intracranial hypertension in men. Neurology. 2009; 72:304309.

9. Ball AK, Clarke CE. Idiopathic intracranial hypertension. Lancet Neurol. 2006; 5:433-442.

10. A.C Onwuchekwa*, C.N. Nwankwo, E.N. ChappJumbo.A 14-year-old Nigerian female with idiopathic intracranial hypertension (Pseudotumor cerebri or benign intracranial hypertension) $\mathrm{Afr}$ Health sci.2002 Dec; 2(3):124-126

11. KC Phillips*and PC Clarke-Farr.Case report:Benign intracranial hypertension diagnosed with bilateral papilloedema Afr Optom 2013 72(2) 97-101

12. Ofori-Asenso R., Agyeman AA, Laar A., Boateng D. Overweight and obesity epidemic in Ghana-a systematic review and meta-analysis. BMC Public Health.2016;16:1239
13. Daniels AB, Liu GT, Volpe NJ, Galetta SL, Moster ML, Newman NJ, Biousse V, Lee AG, Wall M, Kardon R, Acierno MD, Corbett JJ, Maguire MG, Balcer LJ. Profiles of obesity, weight gain, and quality of life in idiopathic intracranial hypertension (pseudotumor cerebri). Am J Ophthalmol. 2007; 143:635-641.

14. Rangwala LM, Liu GT. Pediatric idiopathic intracranial hypertension. Surv Ophthalmol. 2007; 52:597-617.

15. Bruce BB, Kedar S, Van Stavern GP, Corbett JJ, Newman NJ, Biousse V. Atypical idiopathic intracranial hypertension: normal BMI and older patients. Neurology. 2010

16. Balcer LJ, Liu GT, Forman $\mathrm{S}$ et al. Idiopathic intracranial hypertension: relation of age and obesity in children. Neurology. 1999; 52(4):870872.

17. J Lochhead, J. S Elston. Doxycycline induced intracranial hypertension. BMJ 2003 Mar 22; 326(7390)

18. Warner JE, Bernstein PS, Yemelyanov A, Alder SC, Farnsworth ST, Digre KB. Vitamin A in the Cerebrospinal fluid of patients with and without idiopathic intracranial hypertension. Ann Neurol. 2002; 52:647-650

19. Warner JE, Larson AJ, Bhosale P, Digre KB, Henley C, Alder SC, Katz BJ, Bernstein PS. Retinol binding Protein and retinol analysis in cerebrospinal fluid and serum of patients with and without

Idiopathic intracranial hypertension. $J$ Neuroophthalmol. 2007; 27:258-262.

20. Digre KB, Varner MW, Corbett JJ. Pseudotumor cerebri and pregnancy. Neurology. 1984; 34:721729.

21. Ireland B, Corbett JJ, Wallace RB. The search for causes of idiopathic intracranial hypertension: a Preliminary case-control study. Arch Neurol. 1990; 47:315-320.

22. Wall M, George D. Idiopathic intracranial hypertension: a prospective study of 50 patients. Brain. 1991; 114:155-180.

23. Giuseffi V, Wall M, Siegel PZ, Rojas PB. Symptoms and disease associations in idiopathic intracranial hypertension (pseudotumor cerebri): a case-control study. Neurology. 1991; 41:239-244.

24. Bruce BB, Kedar S, Van Stavern GP, Monaghan D, Acierno MD, Braswell RA, Preechawat P, Corbett JJ, Newman NJ, Biousse V. Idiopathic intracranial hypertension in men. Neurology. 2009; 72:304-309.

25. Friedman DI, Rausch EA. Headache diagnoses in patients with treated idiopathic intracranial hypertension. Neurology. 2002; 58:1551-1553. 
26. Yri HM, Rönnbäck C, Wegener M, et al. The course of headache in idiopathic intracranial hypertension: a 12-month prospective follow-up study. Eur $J$ Neurol. 2014; 21:1458-64.

27. Wall M, Kupersmith MJ, Kieburtz KD, et al, NORDIC Idiopathic Intracranial Hypertension Study Group. The idiopathic intracranial hypertension treatment trial: clinical profile at baseline. JAMA Neurol 2014; 71:693-701

28. Corbett JJ, Savino PJ, Thompson HS, Kansu T, Schatz NJ, Orr LS, Hopson D. Visual loss in Pseudotumor cerebri: follow-up of 57 patients from five to 41 years and a profile of 14 patients with permanent severe visual loss. Arch Neurol. 1982; 39:461-474.

29. Thambisetty M, Lavin PJ, Newman NJ, Biousse V. Fulminant idiopathic intracranial hypertension.Neurology. 2007; 68:229-232.

30. Albrecht P.,Blasberg C.,Ringelstein M. Optical Coherence Tomography for the diagnosis and monitoring of IIH.J Neurol.2017

31. Brodsky MC, Vaphiades M. Magnetic resonance imaging in pseudotumor cerebri. Ophthalmology.1998; 105:1686-1693.

32. Agid R, Farb RI, Willinsky RA, Mikulis DJ, Tomlinson G. Idiopathic intracranial hypertension:the validity of cross-sectional neuroimaging signs. Neuroradiology. 2006; 48:5217.
33. Newborg B. Pseudotumor cerebri treated by rice reduction diet. Arch Intern Med. 1974; 133:802-807.

34. Johnson LN, Krohel GB, Madsen RW, March GA Jr. The role of weight loss and acetazolamide in the treatment of idiopathic intracranial hypertension (pseudotumor cerebri). Ophthalmology.1998; 105:2313-2317.

35. Kupersmith MJ, Gamell L, Turbin R, Peck V, Spiegel P, Wall M. Effects of weight loss on the course of idiopathic intracranial hypertension in women. Neurology. 1998; 50:1094-1098.

36. Wong R, Madill SA, Pandey P, Riordan-Eva P. Idiopathic intracranial hypertension: the association between weight loss and the requirement for systemic treatment. BMC Ophthalmol.2007; 7:15.

37. Piper RJ, Kalyvas AV, Young AM, et al. Interventions for idiopathic intracranial hypertension. Cochrane Database Syst Rev 2015; 8:CD003434.

38. Rosenberg ML, Corbett JJ, Smith C, Goodwin J, Sergott R, Savino P, Schatz N. Cerebrospinal fluid diversion procedures in pseudotumor cerebri. Neurology. 1993; 43:1071-1072.

39. Eggenberger ER, Miller NR, Vitale S. Lumboperitoneal shunt for the treatment of pseudotumor cerebri. Neurology. 1996; 46:15241530.

Copyright $($ C The Author(s). This is an Open Access article under the CC BY license. 